\title{
STATISTICAL ANALYSIS OF VARIOUS SUB-SYSTEMS OF PANEL PRODUCTION SYSTEM IN UNDERGROUND COAL MINE
}

\author{
Md. Soyeb Alam ${ }^{1}$ \\ ${ }^{1}$ Assistant Professor, Department of Mining Engineering, Indian School of Mines, Dhanbad-826004, Jharkhand, India, \\ multisoyeb2004@gmail.com
}

\begin{abstract}
System Analysis Approach has been applied in the present field based research paper for a deep and gassy coal mine of Jharia coal fields. The working panel was considered as a System, splitted into various sub-Systems. The Sub-Systems were statistically analyzed in terms of frequency distribution (after prolonged field observations). The Mean, Median, Mode, Standard deviation, Variance and co-efficient of variation were calculated. Among the Mean, Median and Mode, Mean was considered for Capacity (in terms of production) calculation of various Sub-systems
\end{abstract}

\section{INTRODUCTION}

The Supremacy of Coal as a Prime and secured source of energy is unlikely to be challenged in foreseeable future particularly in Indian context. The demands estimates of 620 million ton and 780 million ton during the terminal phases of XI and XII plan respectively also substantiate the larger dependence on coal (Chaoji, 2002 ; Rai et al, 2005). To cater the projected demand targets, the Indian coal industry needs be geared up for accelerated growth in terms of production as well as productivity. The open cast mining, with state of art mechanization is likely to become uneconomical beyond a certain depth, as the open cast technology and equipment have already reached a stage of plateau beyond which further growth is mostly unforeseen (Rai, 2001). Karmakar (1996) has also expressed that production of coal will depend more on underground mine as against present predominance of surface mines. Looking from these standpoints, the Industry may be compelled to increase its share of coal production from underground mines in forth coming years, particularly for deep seated reserves which are vastly untapped at this point of time.

At present, the underground coal mines of our country are generally stricken with problem of low production and productivity (sachdev, 1993 \& Mathur, 1999). This is largely because of the fact that production planning for the underground coal mines is a cumbersome process which calls for sound understanding of various underlying operations to critically assess the sensitive interrelationships amongst them (Mukhopadhaya et al, 1993). Hence, immediate attention and investigation into the performance and appropriate planning process for the underground mines is imperative as also pointed by Bhattacherjee et al, 1996.
The system anlysis based approach appears to offer rationalized solution to solving the complex and intricate production planning from underground mines. In this approach the entire mine (or even a part of the mine) may be considered as system, the capacity of which is dependent on its related sub-systems (Ray et al, 1978). A proper understanding of any system and its related sub-systems could provide intriguing facts in order to practice of this approach in a large underground coal mine which falls in prime coking coal zone of Jharia Coal fields, Dhanbad, India.

\section{AIMS OF THE STUDY:}

As already stated, the present research paper aims at deploying the System Analysis approach for large underground Coal Mine of Jharia Coal Fields. The specific aims of the present work are enumerated as

1. To study the Panel as a system and to identify the important sub-systems for the panel

2. To Critically analysed the various sub-systems Statistically.

\section{RELEVANT DETAILS OF THE MINE \& MINE}

\section{WORKINGS:}

The present Research work was undertaken in a large, privately owned underground 'Mine-A', Jharia Coal Field. One seam, namely XI was being worked in the mine. The bore hole section of the Mine-A has been shown in fig.1.The salient geo-mining details of the Coal seam is as per table $1 \&$ details of panels working as per table 2 . 
Table 1: Salient geo-mining details of the Coal seam

\begin{tabular}{|c|c|}
\hline ITEM & XI SEAM \\
\hline Av. thickness & $7.31 \mathrm{~m}$ \\
\hline Av. depth & $400 \mathrm{~m}$ \\
\hline Av. dip & 1in 7 \\
\hline Dip direction & S76 $50^{\prime} \mathrm{W}$ \\
\hline Shape of seam & Basin like \\
\hline Degree of gassiness & Degree II \\
\hline R.M.R. Value & Highly gaulted with Intrusion \\
\hline Geological features & 3 shaft \\
\hline Mode of entry & CVS \\
\hline Type of ventilation & Through incline by Belt \\
Coal transportation &
\end{tabular}

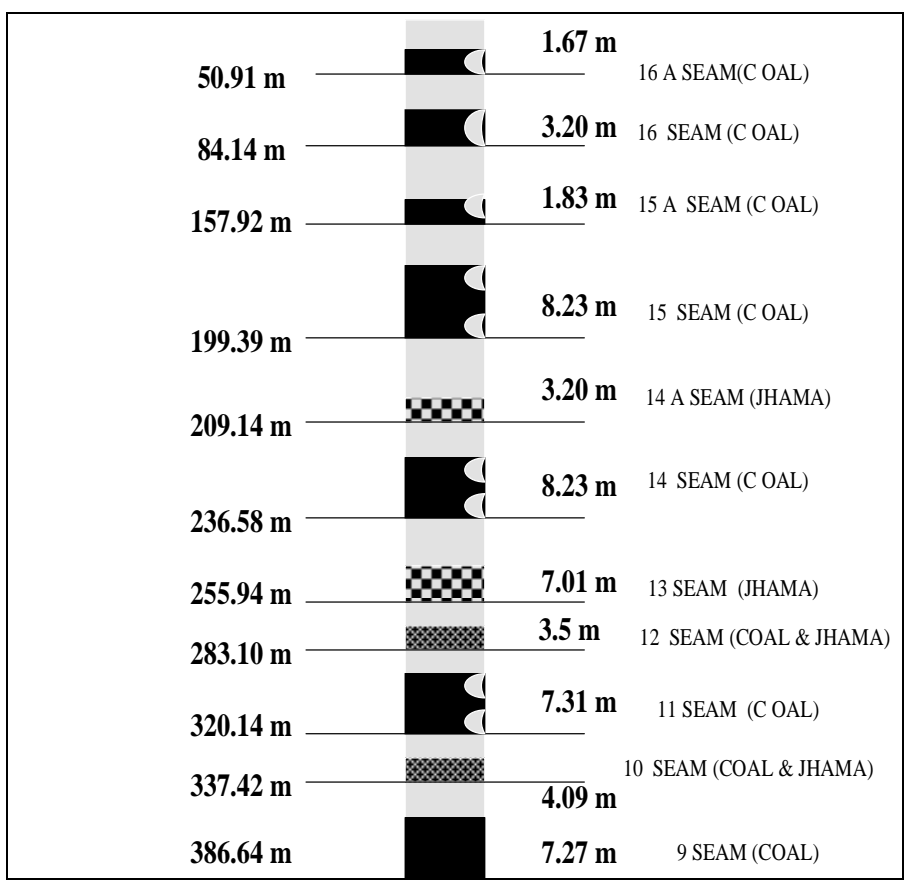

Fig 1: Bore hole section at 9 pit 'Mine-A'

Table 2: Details of panel working in the Mine-A

\begin{tabular}{|c|c|c|c|c|c|c|}
\hline $\begin{array}{l}\text { Panel } \\
\text { Name }\end{array}$ & $\begin{array}{l}\text { Minimum } \\
\text { Avg.depth } \\
\text { (m) }\end{array}$ & $\begin{array}{c}\text { Avg. } \\
\text { pillar } \\
\text { size }(\mathbf{m})\end{array}$ & $\begin{array}{c}\text { Gallery Size }(\mathbf{m}) \\
(\mathbf{w} \times \mathbf{h})\end{array}$ & $\begin{array}{l}\text { Coal preparation } \\
\text { method }\end{array}$ & Loading & Transport \\
\hline $\begin{array}{l}\text { Panel- } \\
11 / 10-S\end{array}$ & 370 & $45 \times 45$ & $\begin{array}{c}4.2 \times 3.0, \\
4.8 \times 3.0(\text { slice })\end{array}$ & $\begin{array}{l}\text { Bord \&Pillar (dep., } \\
\text { bottom sec.) }\end{array}$ & SDL & Conveyor \\
\hline $\begin{array}{l}\text { Panel- } \\
11 / 7-S\end{array}$ & 400 & $45 \times 45$ & $\begin{array}{c}4.2 \times 2.8 \\
4.8 \times 2.8 \text { (slice) }\end{array}$ & $\begin{array}{l}\text { Bord \& Pillar (dev. \& } \\
\text { dep., top sec.) }\end{array}$ & SDL & Conveyor \\
\hline $\begin{array}{l}\text { Panel- } \\
\text { 11/3-S }\end{array}$ & 400 & $45 \times 45$ & $\begin{array}{c}4.2 \times 2.7 \\
4.8 \times 2.7 \text { (slice) }\end{array}$ & $\begin{array}{l}\text { Bord \& Pillar (dep., } \\
\text { top sec.) }\end{array}$ & manual & Conveyor \\
\hline $\begin{array}{l}\text { Panel- } \\
11 / 9-S\end{array}$ & 325 & $45 \times 45$ & $\begin{array}{c}4.2 \times 2.7 \\
4.8 \times 2.7 \text { (slice) }\end{array}$ & $\begin{array}{c}\text { Bord \& Pillar (dev. \& } \\
\text { dep., bottom \& top } \\
\text { sec. both) }\end{array}$ & SDL & Conveyor \\
\hline
\end{tabular}

\section{BRIEF DESCRIPTION OF COAL WINNING IN THE STUDY PANEL}

There were three semi-mechanised panels namely, panel '11/7-S', '11/10-S' \& '11/9-S' and one manual panel namely panel ' $11 / 3-S$ ' in the given mine. The study was conducted in the semi-mechanised panel $11 / 7-\mathrm{S}$ in the mine. The description of the coal winning in the study panel as follows:

Bottom section of panel 11/7-S had been developed and depillared upto $3.0 \mathrm{~m}$ height by bord and pillar (B/P) method of working in conjunction with hydraulic sand stowing. some portion of bottom section had been left for the purpose of sumping. Depillaring in bottom section had been done by splitting pillar into four parts. After leaving a parting of almost $1.5 \mathrm{~m}$, top section $2.8 \mathrm{~m}$ was being developed \& depillared simultaneously by B/P method of working. Top section depillaring by means of modified depillaring method in which taking dip-rise slice simultaneously then judicial extraction of rib in conjunction with hydraulic sand stowing

Panel consisted of 3 SDLs, (one SDL for development and two SDLs for depillaring) loading coal on separate light duty chain conveyor (LDCC). Coal broken by drilling \& blasting 
was loaded on LDCC by means of side discharge loader (SDL) with bucket capacity of $1.5 \mathrm{cu} . \mathrm{m}$. face conveyor discharged the coal on the belt conveyor to be carried out bye through a system of belts up to the coal washery located on surface. The galleries, splits and slices were supported systematically by the roof bolts having yield load of 5 tonne.

\section{RESEARCH METHODOLOGY}

The aforesaid concept of breaking a mine system into subsystems for finer analysis, has been adopted in the present case study, considering Panel as a system. According the major sub-systems were formulated as given in fig. 2 .

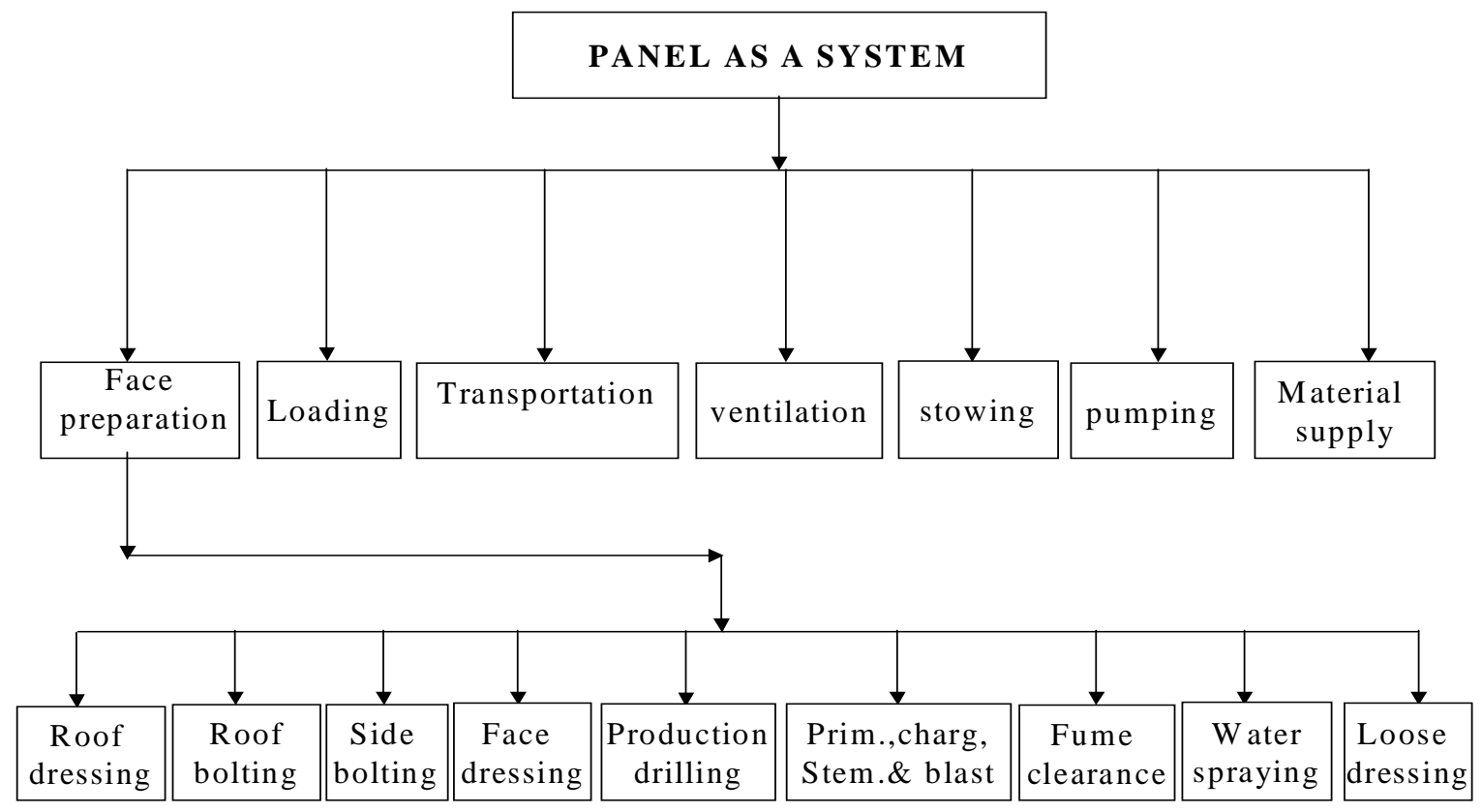

Fig. 2: Formulated systematic proposed model for Present working

\section{FIELD OBSERVATIONS:}

\subsection{Time and Motion Study of Various Sub-Systems:}

As per the formulated system model (fig.2) the time \& motion studies were conducted at field scale for various subsystems. The statistical data pertaining to the time \& motion studies of various sub-systems such as, face preparation (roof dressing, roof bolting, side bolting etc.) loading etc. in statistical format is tabulated in tables 3 to 20. Based on the statistical tables the mean, median, mode, standard deviation $\&$ co-efficient of variation for face preparation $\&$ loading and mean (average) for stowing \& ventilation sub-systems.

Table 3: Frequency distribution of roof dressing per face (minute)

\begin{tabular}{|c|c|c|c|c|c|c|}
\hline $\begin{array}{c}\text { Class } \\
\text { mark } \\
(\mathbf{x}) \\
\end{array}$ & $\begin{array}{c}\text { Frequency } \\
\text { (f) }\end{array}$ & $f x$ & $\begin{array}{c}\text { Cumulative } \\
\text { frequency } \\
\text { (c.f) }\end{array}$ & $\begin{array}{c}d=x-A \\
=x-20.18\end{array}$ & $d^{2}$ & $\mathbf{f d}^{2}$ \\
\hline 14 & 11 & 154 & 11 & -6.18 & 38.19 & 420.12 \\
\hline 15 & 18 & 270 & 29 & -5.18 & 26.83 & 482.98 \\
\hline 16 & 7 & 112 & 36 & -4.18 & 17.47 & 122.31 \\
\hline 17 & 3 & 51 & 39 & -3.18 & 10.11 & 30.34 \\
\hline 18 & 3 & 54 & 42 & -2.18 & 4.75 & 14.26 \\
\hline 19 & 2 & 38 & 44 & -1.18 & 1.39 & 2.78 \\
\hline 20 & 2 & 40 & 46 & -.18 & .03 & .06 \\
\hline
\end{tabular}




\begin{tabular}{|c|c|c|c|c|c|c|}
\hline 50 & 1 & 50 & 47 & 29.82 & 889.23 & 889.23 \\
\hline 55 & 1 & 55 & 48 & 34.82 & 1212.43 & 1212.43 \\
\hline 60 & 1 & 60 & 49 & 39.82 & 1585.63 & 1585.63 \\
\hline 70 & 1 & 70 & 50 & 49.82 & 2482 & 2482 \\
\hline 75 & 1 & 75 & 51 & 54.82 & 3005.23 & 3005.23 \\
\hline & $\sum \mathrm{f}=51$ & $\sum \mathrm{fx}=1029$ & & & & $\sum \mathrm{fd}^{2}=10247.37$ \\
\hline
\end{tabular}
$\begin{aligned} \operatorname{Mean}(\mathrm{A})=\sum \mathrm{fx} / \sum \mathrm{f}=1029 / 51 & =20.18, \operatorname{Median}(\mathrm{M})=15, \\ & =1 \text { Mode }(\mathrm{Mo})=15,\end{aligned}$

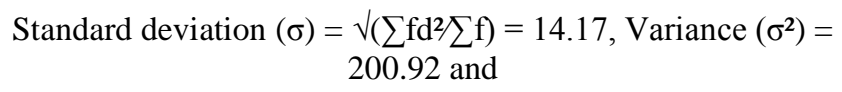

Co-efficient of variation $=(\sigma / \mathrm{A}) \times 100=70.24 \%$

Table 4: Frequency distribution of roof bolting per bolt (minute)

\begin{tabular}{|c|c|c|c|c|c|c|c|}
\hline $\begin{array}{c}\text { Class } \\
\text { interval }\end{array}$ & $\begin{array}{c}\text { Class } \\
\text { mark (x) }\end{array}$ & $\begin{array}{c}\text { Frequency } \\
(\mathbf{f})\end{array}$ & $\mathbf{f x}$ & $\mathbf{c . f}$ & $\begin{array}{c}\mathbf{d}=\mathbf{x}-\mathbf{A} \\
\mathbf{x}-\mathbf{7 . 3 2}\end{array}$ & $\mathbf{d}^{\mathbf{2}}$ & $\mathbf{f d}^{\mathbf{2}}$ \\
\hline $4-6$ & 5 & 40 & 200 & 40 & -2.32 & 5.38 & 215.29 \\
\hline $6-8$ & 7 & 118 & 826 & 158 & -.32 & .10 & 12.08 \\
\hline $8-10$ & 9 & 20 & 180 & 178 & 1.68 & 2.82 & 56.45 \\
\hline $10-12$ & 11 & 2 & 22 & 180 & 3.68 & 13.54 & 27.08 \\
\hline $12-14$ & 13 & 2 & 26 & 182 & 5.68 & 32.26 & 64.52 \\
\hline $14-16$ & 15 & 5 & 75 & 187 & 7.68 & 58.98 & 294.91 \\
\hline $16-18$ & 17 & 3 & 51 & 190 & 9.68 & 93.7 & 281.10 \\
\hline $18-20$ & 19 & 1 & 19 & 191 & 11.68 & 136.42 & 136.42 \\
\hline & & $\sum \mathrm{f}=191$ & $\sum \mathrm{fx}=1399$ & & & & $\sum \mathrm{fd}^{2}=1087.85$ \\
\hline
\end{tabular}

$\operatorname{Mean}(\mathrm{A})=\sum \mathrm{fx} / \sum \mathrm{f}=1399 / 191=7.32, \operatorname{Median}(\mathrm{M})=6.94$,

$$
\text { Mode }(\mathrm{Mo})=6.88 \text {, }
$$

Standard deviation $(\sigma)=\sqrt{ }\left(\sum \mathrm{fd}^{2} / \sum \mathrm{f}\right)=2.38$, Variance $\left(\sigma^{2}\right)=$ 5.69 and

Table 5: Frequency distribution of side bolting per face (minute)

\begin{tabular}{|c|c|c|c|c|c|c|c|}
\hline $\begin{array}{c}\text { Class } \\
\text { interval }\end{array}$ & $\begin{array}{c}\text { Class } \\
\text { mark } \\
(\mathbf{x})\end{array}$ & $\begin{array}{c}\text { Frequency } \\
(\mathbf{f})\end{array}$ & $\mathbf{f x}$ & $\mathbf{c . f}$ & $\begin{array}{c}\mathbf{d}=\mathbf{x}-\mathbf{A} \\
\mathbf{x}-\mathbf{3 . 9 7}\end{array}$ & $\mathbf{d}^{2}$ & $\mathbf{f d}^{\mathbf{2}}$ \\
\hline $2.5-3.0$ & 2.75 & 6 & 16.5 & 6 & -1.22 & 1.490 & 8.93 \\
\hline $3.0-3.5$ & 3.25 & 5 & 16.25 & 11 & -0.72 & 0.52 & 2.59 \\
\hline $3.5-4.0$ & 3.75 & 17 & 63.75 & 28 & -0.22 & 0.0484 & 0.82 \\
\hline $4.0-4.5$ & 4.25 & 6 & 25.5 & 34 & 0.5 & 0.25 & 1.5 \\
\hline $4.5-5.0$ & 4.75 & 13 & 61.75 & 47 & 0.78 & 0.61 & 7.91 \\
\hline $5.0-5.5$ & 5.25 & 1 & 5.25 & 48 & 1.28 & 1.64 & 1.64 \\
\hline $5.5-6.0$ & 5.75 & 1 & 5.75 & 49 & 1.78 & 3.17 & 3.17 \\
\hline & & $\sum \mathrm{f}=49$ & $\sum \mathrm{fx}=194.75$ & & & & $\sum \mathrm{fd}^{2}=26.56$ \\
\hline
\end{tabular}

Mean $(A)=\sum f x / \sum f=194.75 / 49=3.97, \operatorname{Median}(M)=$ 3.89 , Mode $(\mathrm{Mo})=3.76$,
Standard deviation $(\sigma)=\sqrt{ }\left(\sum \mathrm{fd}^{2} / \sum \mathrm{f}\right)=0.736$, Variance $\left(\sigma^{2}\right)=$ 0.542 and

Co-efficient of variation $=(\sigma / \mathrm{A}) \times 100=18.53 \%$ 
Table 6: Frequency distribution of face dressing per face (minute)

\begin{tabular}{|c|c|c|c|c|c|c|c|}
\hline $\begin{array}{c}\text { Class } \\
\text { interval }\end{array}$ & $\begin{array}{c}\text { Class } \\
\text { mark } \\
(\mathbf{x})\end{array}$ & $\begin{array}{c}\text { Frequency } \\
(\mathbf{f})\end{array}$ & $\mathbf{f x}$ & $\mathbf{c . f}$ & $\begin{array}{c}\mathbf{d}=\mathbf{x}-\mathbf{A} \\
\mathbf{x}-\mathbf{8 . 8 4}\end{array}$ & $\mathbf{d}^{\mathbf{2}}$ & $\mathbf{f d}^{\mathbf{2}}$ \\
\hline $6-7$ & 6.5 & 10 & 65 & 10 & -2.34 & 5.475 & 54.75 \\
\hline $7-8$ & 7.5 & 14 & 105 & 24 & -1.34 & 1.795 & 25.14 \\
\hline $8-9$ & 8.5 & 8 & 68 & 32 & -0.34 & 0.115 & .92 \\
\hline $9-10$ & 9.5 & 8 & 76 & 40 & 0.66 & 0.435 & 3.48 \\
\hline $10-11$ & 10.5 & 0 & 0 & 40 & 1.66 & 2.755 & 0 \\
\hline $11-12$ & 11.5 & 0 & 0 & 40 & 2.66 & 7.075 & 0 \\
\hline $12-13$ & 12.5 & 0 & 0 & 40 & 3.66 & 13.395 & 0 \\
\hline $13-14$ & 13.5 & 2 & 27 & 42 & 4.66 & 21.72 & 43.43 \\
\hline $14-15$ & 14.5 & 3 & 43.5 & 45 & 5.66 & 32.03 & 96.11 \\
\hline $15-16$ & 16.5 & 2 & 31.0 & 47 & 6.66 & 44.75 & 88.71 \\
\hline & & $\sum \mathrm{f}=47$ & $\sum \mathrm{fx}=415.5$ & & & & $\sum \mathrm{fd}^{2}=312.54$ \\
\hline
\end{tabular}

Standard deviation $(\sigma)=\sqrt{ }\left(\sum \mathrm{fd}^{2} / \sum \mathrm{f}\right)=2.578$, Variance $\left(\sigma^{2}\right)=$

$\operatorname{Mean}(\mathrm{A})=\sum \mathrm{fx} / \sum \mathrm{f}=415.5 / 47=8.84, \operatorname{Median}(\mathrm{M})=7.96$, 6.65 and

Mode $(\mathrm{Mo})=7.4$

Co-efficient of variation $=(\sigma / \mathrm{A}) \times 100=29.16 \%$

Table 7: Frequency distribution of production drilling per hole (second)

\begin{tabular}{|c|c|c|c|c|c|c|c|}
\hline $\begin{array}{c}\text { Class } \\
\text { interval }\end{array}$ & $\begin{array}{c}\text { Class } \\
\text { mark } \\
(\mathbf{x})\end{array}$ & $\begin{array}{c}\text { Frequency } \\
(\mathbf{f})\end{array}$ & $\mathbf{f x}$ & $\mathbf{c . f}$ & $\begin{array}{c}\mathbf{d}=\mathbf{x}-\mathbf{A} \\
\mathbf{x}-\mathbf{8 0}\end{array}$ & $\mathbf{d}^{2}$ & $\mathbf{f d}^{2}$ \\
\hline $0-20$ & 10 & 1 & 10 & 1 & -70 & 4900 & 4900 \\
\hline $20-40$ & 30 & 9 & 570 & 10 & -50 & 2500 & 22500 \\
\hline $40-60$ & 50 & 19 & 950 & 29 & -30 & 900 & 17100 \\
\hline $60-80$ & 70 & 51 & 3570 & 80 & -10 & 100 & 5100 \\
\hline $80-100$ & 90 & 26 & 2340 & 106 & 10 & 100 & 2600 \\
\hline $100-120$ & 110 & 17 & 1870 & 123 & 30 & 900 & 15300 \\
\hline $120-140$ & 130 & 68 & 1040 & 131 & 50 & 2500 & 20000 \\
\hline $140-160$ & 150 & 2 & 300 & 133 & 70 & 4900 & 9800 \\
\hline & & $\sum \mathrm{f}=133$ & $\sum \mathrm{fx}=10650$ & & & & $\sum \mathrm{fd}^{2}=97300$ \\
\hline
\end{tabular}

$\operatorname{Mean}(\mathrm{A})=\sum \mathrm{fx} / \sum \mathrm{f}=10650 / 133=80, \operatorname{Median}(\mathrm{M})=74.7$, Mode $(\mathrm{Mo})=71.23$,
Standard deviation $(\sigma)=\sqrt{ }\left(\sum \mathrm{fd}^{2} / \sum \mathrm{f}\right)=27.04$, Variance $\left(\sigma^{2}\right)=$ 731.57 and

Co-efficient of variation $=(\sigma / \mathrm{A}) \times 100=33.7 \%$

Table 8: Frequency distribution of preparing sand capsule, 36 capsule with 3 men (minute)

\begin{tabular}{|c|c|c|c|c|c|c|}
\hline $\begin{array}{c}\text { Class } \\
\operatorname{mark}(\mathbf{x})\end{array}$ & $\begin{array}{c}\text { Frequency } \\
\text { (f) }\end{array}$ & $\mathbf{f x}$ & c.f & $\begin{array}{c}d=x-A \\
=x-17.44\end{array}$ & $d^{2}$ & $\mathrm{fd}^{2}$ \\
\hline 12 & 1 & 12 & 1 & -5.44 & 29.59 & 29.59 \\
\hline 13 & 3 & 39 & 4 & -4.44 & 19.71 & 59.14 \\
\hline 14 & 4 & 56 & 8 & -3.44 & 11.83 & 47.33 \\
\hline 15 & 6 & 90 & 14 & -2.44 & 5.95 & 35.72 \\
\hline 16 & 4 & 64 & 18 & -1.44 & 2.07 & 8.29 \\
\hline 17 & 3 & 51 & 21 & -.44 & 0.19 & .58 \\
\hline 18 & 3 & 54 & 24 & 1.44 & 2.07 & 6.21 \\
\hline 30 & 1 & 30 & 25 & 12.56 & 157.75 & 157.75 \\
\hline
\end{tabular}




\begin{tabular}{|c|c|c|c|c|c|c|}
\hline 35 & 1 & 35 & 26 & 17.56 & 308.35 & 308.35 \\
\hline 40 & 1 & 40 & 27 & 22.56 & 508.95 & 508.95 \\
\hline & $\sum \mathrm{f}=27$ & $\sum \mathrm{fx}=471$ & & & & $\sum \mathrm{fd}^{2}=1161.91$ \\
\hline
\end{tabular}

$\operatorname{Mean}(\mathrm{A})=\sum \mathrm{fx} / \sum \mathrm{f}=471 / 27=17.44, \operatorname{Median}(\mathrm{M})=15$, Mode $(\mathrm{Mo})=15$,
Standard deviation $(\sigma)=\sqrt{ }\left(\sum \mathrm{fd}^{2} / \sum \mathrm{f}\right)=6.56$, Variance $\left(\sigma^{2}\right)=$ 43.03 and

Co-efficient of variation $=(\sigma / \mathrm{A}) \times 100=37.61 \%$

Table 9: Frequency distribution of charging and blasting per face (minute)

\begin{tabular}{|c|c|c|c|c|c|c|c|}
\hline $\begin{array}{c}\text { Class } \\
\text { interval }\end{array}$ & $\begin{array}{c}\text { Class } \\
\text { mark } \\
(\mathbf{x})\end{array}$ & $\begin{array}{c}\text { Frequency } \\
(\mathbf{f})\end{array}$ & $\mathbf{f x}$ & $\mathbf{c . f}$ & $\begin{array}{c}\mathbf{d}=\mathbf{x}-\mathbf{A} \\
\mathbf{x}-\mathbf{3 6}\end{array}$ & $\mathbf{d}^{\mathbf{2}}$ & $\mathbf{f d}^{\mathbf{2}}$ \\
\hline $29-31$ & 30 & 7 & 210 & 7 & -6 & 36 & 252 \\
\hline $31-33$ & 32 & 4 & 128 & 11 & -4 & 16 & 64 \\
\hline $33-35$ & 34 & 7 & 238 & 18 & -2 & 4 & 8 \\
\hline $35-37$ & 36 & 1 & 36 & 19 & 0 & 0 & 0 \\
\hline $37-39$ & 38 & 6 & 228 & 25 & 2 & 4 & 24 \\
\hline $39-41$ & 40 & 4 & 160 & 29 & 4 & 16 & 64 \\
\hline $41-43$ & 42 & 3 & 126 & 32 & 6 & 36 & 108 \\
\hline $43-45$ & 44 & 2 & 88 & 34 & 8 & 64 & 128 \\
\hline $45-47$ & 46 & 1 & 46 & 35 & 10 & 100 & 100 \\
\hline & & $\sum \mathrm{f}=35$ & $\sum \mathrm{fx}=1260$ & & & & $\sum \mathrm{fd}^{2}=768$ \\
\hline
\end{tabular}

Mean $(A)=\sum f x / \sum f=1260 / 35=36, \operatorname{Median}(M)=34.85$, Mode $(\mathrm{Mo})=$
33.67, Standard deviation $(\sigma)=\sqrt{ }\left(\sum \mathrm{fd}^{2} / \sum \mathrm{f}\right)=4.68$, Variance $\left(\sigma^{2}\right)=21.94$ and

Co-efficient of variation $=(\sigma / \mathrm{A}) \times 100=13 \%$

Table 10: Frequency distribution of fume clearance per face (minute)

\begin{tabular}{|c|c|c|c|c|c|c|c|}
\hline $\begin{array}{c}\text { Class } \\
\text { interval }\end{array}$ & $\begin{array}{c}\text { Class } \\
\text { mark } \\
(\mathbf{x})\end{array}$ & $\begin{array}{c}\text { Frequency } \\
(\mathbf{f})\end{array}$ & $\mathbf{f x}$ & $\mathbf{c . f}$ & $\begin{array}{c}\mathbf{d}=\mathbf{x}-\mathbf{A} \\
\mathbf{x}-\mathbf{4 . 7 8}\end{array}$ & $\mathbf{d}^{\mathbf{2}}$ & $\mathbf{f d}^{\mathbf{2}}$ \\
\hline $3.5-4.0$ & 3.75 & 2 & 7.5 & 2 & -1.03 & 1.06 & 2.12 \\
\hline $4.0-4.5$ & 4.25 & 12 & 51 & 14 & -0.53 & 0.28 & 3.37 \\
\hline $4.5-5.0$ & 4.75 & 13 & 61.75 & 27 & -0.03 & .0009 & 0.0117 \\
\hline $5.0-5.5$ & 5.25 & 6 & 31.5 & 33 & 0.47 & .22 & 1.325 \\
\hline $5.5-6.0$ & 5.75 & 4 & 23 & 37 & 0.97 & 0.94 & 3.76 \\
\hline $6.0-6.5$ & 6.25 & 0 & 0 & 37 & 1.47 & 2.16 & 0 \\
\hline $6.5-7.0$ & 6.75 & 1 & 6.75 & 38 & 1.97 & 3.88 & 3.88 \\
\hline & & $\sum \mathrm{f}=38$ & $\sum \mathrm{fx}=181.5$ & & & & $\sum \mathrm{fd}^{2}=14.46$ \\
\hline
\end{tabular}

$\operatorname{Mean}(\mathrm{A})=\sum \mathrm{fx} / \sum \mathrm{f}=181.5 / 38=4.78, \operatorname{Median}(\mathrm{M})=$ 4.69 , Mode $(\mathrm{Mo})=4.56$,
Standard deviation $(\sigma)=\sqrt{ }\left(\sum \mathrm{fd}^{2} / \sum \mathrm{f}\right)=0.616$, Variance $\left(\sigma^{2}\right)=$ 0.381 and

Co-efficient of variation $=(\sigma / \mathrm{A}) \times 100=12.88 \%$ 
Table 11: Frequency distribution of time study of water spraying and loose dressing per face (minute)

\begin{tabular}{|c|c|c|c|c|c|c|c|}
\hline $\begin{array}{c}\text { Class } \\
\text { interval }\end{array}$ & $\begin{array}{c}\text { Class } \\
\text { mark } \\
(\mathbf{x})\end{array}$ & $\begin{array}{c}\text { Frequency } \\
(\mathbf{f})\end{array}$ & $\mathbf{f x}$ & $\mathbf{c . f}$ & $\begin{array}{c}\mathbf{d}=\mathbf{x}-\mathbf{A} \\
\mathbf{x}-\mathbf{1 7 . 9 8}\end{array}$ & $\mathbf{d}^{2}$ & $\mathbf{f d}^{2}$ \\
\hline $14-15$ & 14.5 & 2 & 29 & 2 & -3.48 & 12.11 & 24.22 \\
\hline $15-16$ & 15.5 & 3 & 46.5 & 5 & -2.48 & 6.15 & 18.45 \\
\hline $16-17$ & 16.5 & 2 & 33 & 7 & -1.48 & 2.19 & 4.38 \\
\hline $17-18$ & 17.5 & 6 & 105 & 13 & -0.48 & .23 & 1.38 \\
\hline $18-19$ & 18.5 & 6 & 111 & 19 & 0.58 & .27 & 1.68 \\
\hline $19-20$ & 19.5 & 4 & 78 & 23 & 1.58 & 2.31 & 9.24 \\
\hline $20-21$ & 20.5 & 3 & 61.5 & 26 & 2.52 & 6.35 & 19.05 \\
\hline $21-22$ & 21.5 & 1 & 21.5 & 27 & 3.52 & 12.39 & 12.39 \\
\hline & & $\sum \mathrm{f}=27$ & $\sum \mathrm{fx}=485.5$ & & & & $\sum \mathrm{fd}^{2}=295.32$ \\
\hline
\end{tabular}

Mean $(\mathrm{A})=\sum \mathrm{fx} / \sum \mathrm{f}=485.5 / 27=17.98, \operatorname{Median}(\mathrm{M})=$
$18.08, \operatorname{Mode}(\mathrm{Mo})=18$ $\begin{aligned} \text { Standard deviation }(\sigma)= & \sqrt{ }\left(\sum \mathrm{fd}^{2} / \sum \mathrm{f}\right)=3.31, \text { Variance }\left(\sigma^{2}\right)= \\ & 10.93 \text { and }\end{aligned}$

Co-efficient of variation $=(\sigma / \mathrm{A}) \times 100=18.41 \%$

Table 12: Frequency distribution of loading cycle time (second) at lead up to 5 metres

\begin{tabular}{|c|c|c|c|c|c|c|c|}
\hline $\begin{array}{c}\text { Class } \\
\text { interval }\end{array}$ & $\begin{array}{c}\text { Class } \\
\text { mark } \\
(\mathbf{x})\end{array}$ & $\begin{array}{c}\text { Frequency } \\
(\mathbf{f})\end{array}$ & $\mathbf{f x}$ & $\mathbf{c . f}$ & $\begin{array}{c}\mathbf{d}=\mathbf{x}-\mathbf{A} \\
\mathbf{x}-\mathbf{5 1 . 1 2}\end{array}$ & $\mathbf{d}^{2}$ & $\mathbf{f d}^{2}$ \\
\hline $35-40$ & 37.5 & 8 & 300 & 8 & -13.62 & 185.50 & 1484.03 \\
\hline $40-45$ & 42.5 & 23 & 977.5 & 31 & -8.62 & 74.30 & 1709.00 \\
\hline $45-50$ & 47.5 & 19 & 902.5 & 50 & -3.62 & 13.10 & 248.98 \\
\hline $50-55$ & 52.5 & 10 & 525 & 60 & 1.38 & 1.90 & 19.04 \\
\hline $55-60$ & 57.5 & 17 & 977.5 & 77 & 6.38 & 40.70 & 691.97 \\
\hline $60-65$ & 62.5 & 9 & 562.5 & 86 & 11.38 & 129.50 & 1165.53 \\
\hline $65-70$ & 67.5 & 5 & 337.5 & 91 & 16.38 & 268.30 & 1341.52 \\
\hline $70-75$ & 72.5 & 2 & 145 & 93 & 21.38 & 457.10 & 914.21 \\
\hline $75-80$ & 77.5 & 1 & 77.5 & 94 & 26.38 & 695.90 & 695.90 \\
\hline & & $\sum \mathrm{f}=94$ & $\sum \mathrm{fx}=4805$ & & & & $\sum \mathrm{fd}^{2}=8270.18$ \\
\hline
\end{tabular}
$\begin{aligned} \text { Mean }(\mathrm{A})= & \sum \mathrm{fx} / \sum \mathrm{f}=4805 / 94=51.12, \operatorname{Median}(\mathrm{M})= \\ & 49.21, \operatorname{Mode}(\mathrm{Mo})=43.95\end{aligned}$ $\begin{aligned} \text { Standard deviation }(\sigma)= & \sqrt{ }\left(\sum \mathrm{fd}^{2} \sum \mathrm{f}\right)=9.38, \text { Variance }\left(\sigma^{2}\right)= \\ & 87.98 \text { and }\end{aligned}$

Co-efficient of variation $=(\sigma / \mathrm{A}) \times 100=18.34 \%$

Table 13: Frequency distribution of loading cycle time (second) at lead 5-10 metres

\begin{tabular}{|c|c|c|c|c|c|c|c|}
\hline $\begin{array}{c}\text { Class } \\
\text { interval }\end{array}$ & $\begin{array}{c}\text { Class } \\
\text { mark } \\
(\mathbf{x})\end{array}$ & $\begin{array}{c}\text { Frequency } \\
(\mathbf{f})\end{array}$ & $\mathbf{f x}$ & $\mathbf{c . f}$ & $\begin{array}{c}\mathbf{d}=\mathbf{x}-\mathbf{A} \\
\mathbf{x}-\mathbf{7 5 . 5 8}\end{array}$ & $\mathbf{d}^{2}$ & $\mathbf{f d}^{2}$ \\
\hline $65-70$ & 67.5 & 15 & 1012.5 & 15 & -8.08 & 65.28 & 979.29 \\
\hline $70-75$ & 72.5 & 17 & 1232.5 & 32 & -3.08 & 9.48 & 161.27 \\
\hline $75-80$ & 77.5 & 3 & 232.5 & 35 & 1.92 & 3.68 & 11.06 \\
\hline $80-85$ & 82.5 & 3 & 247.5 & 38 & 6.92 & 47.88 & 143.66 \\
\hline
\end{tabular}




\begin{tabular}{|c|c|c|c|c|c|c|c|}
\hline $85-90$ & 87.5 & 3 & 262.5 & 41 & 11.92 & 142.08 & 426.24 \\
\hline $90-95$ & 92.5 & 4 & 370 & 45 & 16.92 & 286.28 & 1145.14 \\
\hline $95-100$ & 97.5 & 2 & 195 & 47 & 21.92 & 480.48 & 960.97 \\
\hline & & $\sum \mathrm{f}=47$ & $\sum \mathrm{fx}=3552.5$ & & & & $\sum \mathrm{fd}^{2}=3827.63$ \\
\hline
\end{tabular}

Mean $(\mathrm{A})=\sum \mathrm{fx} / \sum \mathrm{f}=3552.5 / 47=75.58, \operatorname{Median}(\mathrm{M})=$ 72.5 , Mode $(\mathrm{Mo})=70.63$
Standard deviation $(\sigma)=\sqrt{ }\left(\sum \mathrm{fd}^{2} / \sum \mathrm{f}\right)=9.02$, Variance $\left(\sigma^{2}\right)$ $=81.44$ and

Co-efficient of variation $=(\sigma / \mathrm{A}) \times 100=11.94 \%$

Table 14: Frequency distribution of loading cycle time (second) at lead 10-15 metres

\begin{tabular}{|c|c|c|c|c|c|c|c|}
\hline $\begin{array}{c}\text { Class } \\
\text { interval }\end{array}$ & $\begin{array}{c}\text { Class } \\
\text { mark } \\
(\mathbf{x})\end{array}$ & $\begin{array}{c}\text { Frequency } \\
(\mathbf{f})\end{array}$ & $\mathbf{f x}$ & $\mathbf{c . f}$ & $\begin{array}{c}\mathbf{d}=\mathbf{x}-\mathbf{A} \\
\mathbf{x} \mathbf{x}-\mathbf{8 3 . 2 9}\end{array}$ & $\mathbf{d}^{2}$ & $\mathbf{f d}^{\mathbf{2}}$ \\
\hline $70-75$ & 72.5 & 5 & 362.5 & 5 & -10.79 & 116.42 & 582.12 \\
\hline $75-80$ & 77.5 & 7 & 542.5 & 12 & -5.79 & 33.52 & 134.67 \\
\hline $80-85$ & 82.5 & 17 & 1402.5 & 29 & -0.79 & .624 & 10.61 \\
\hline $85-90$ & 87.5 & 9 & 787.5 & 38 & 4.21 & 17.72 & 159.52 \\
\hline $90-95$ & 92.5 & 4 & 370 & 42 & 9.21 & 84.82 & 339.29 \\
\hline $95-100$ & 97.5 & 1 & 97.5 & 43 & 14.21 & 201.92 & 201.92 \\
\hline $100-105$ & 102.5 & 1 & 102.5 & 44 & 19.21 & 369.02 & 369.02 \\
\hline & & $\sum \mathrm{f}=44$ & $\sum \mathrm{fx}=3665$ & & & & $\sum \mathrm{fd}^{2}=1897.15$ \\
\hline
\end{tabular}

Mean $(A)=\sum f x / \sum f=3665 / 44=83.29, \operatorname{Median}(M)=$ 82.94 , Mode $(\mathrm{Mo})=82.77$
Standard deviation $\begin{aligned}(\sigma)= & \sqrt{ }\left(\sum \mathrm{fd}^{2} / \sum \mathrm{f}\right)=6.57, \text { Variance }\left(\sigma^{2}\right) \\ & =43.11\end{aligned}$

And Co-efficient of variation $=(\sigma / \mathrm{A}) \times 100=7.88 \%$

Table 15: Frequency distribution of loading cycle time (second) at lead 15-20 metres

\begin{tabular}{|c|c|c|c|c|c|c|c|}
\hline $\begin{array}{c}\text { Class } \\
\text { interval }\end{array}$ & $\begin{array}{c}\text { Class } \\
\text { mark } \\
(\mathbf{x})\end{array}$ & $\begin{array}{c}\text { Frequency } \\
(\mathbf{f})\end{array}$ & $\mathbf{f x}$ & $\mathbf{c . f}$ & $\begin{array}{c}\mathbf{d}=\mathbf{x}-\mathbf{A} \\
\mathbf{x}-\mathbf{9 9 . 7 4}\end{array}$ & $\mathbf{d}^{2}$ & $\mathbf{f d}^{\mathbf{2}}$ \\
\hline $90-95$ & 92.5 & 17 & 1572.5 & 17 & -7.24 & 52.42 & 891.14 \\
\hline $95-100$ & 97.5 & 14 & 1365 & 31 & -2.24 & 5.02 & 70.25 \\
\hline $100-105$ & 102.5 & 7 & 717.5 & 38 & 2.76 & 7.62 & 53.32 \\
\hline $105-110$ & 107.5 & 5 & 537.5 & 43 & 7.76 & 60.22 & 301.08 \\
\hline $110-115$ & 112.5 & 3 & 337.5 & 46 & 12.76 & 162.82 & 488.45 \\
\hline $115-120$ & 117.5 & 2 & 235 & 48 & 17.76 & 315.42 & 630.83 \\
\hline $120-125$ & 122.5 & 1 & 122.5 & 49 & 22.76 & 518.02 & 518.02 \\
\hline & & $\sum \mathrm{f}=49$ & $\sum \mathrm{fx}=4887.5$ & & & & $\sum \mathrm{fd}^{2}=2453.09$ \\
\hline
\end{tabular}

$\operatorname{Mean}(\mathrm{A})=\sum \mathrm{fx} / \sum \mathrm{f}=4887.5 / 49=99.74, \operatorname{Median}(\mathrm{M})=$ 97.67, Mode $(\mathrm{Mo})=94.25$
Standard deviation $(\sigma)=\sqrt{ }\left(\sum \mathrm{fd}^{2} / \sum \mathrm{f}\right)=7.07$, Variance $\left(\sigma^{2}\right)$ $=50.06$ and

Co-efficient of variation $=(\sigma / \mathrm{A}) \times 100=7.09 \%$ 
Table 16: Frequency distribution of loading cycle time (second) at lead 20-25 metres

\begin{tabular}{|c|c|c|c|c|c|c|c|}
\hline $\begin{array}{c}\text { Class } \\
\text { interval }\end{array}$ & $\begin{array}{c}\text { Class } \\
\text { mark } \\
(\mathbf{x})\end{array}$ & $\begin{array}{c}\text { Frequency } \\
(\mathbf{f})\end{array}$ & $\mathbf{f x}$ & $\mathbf{c . f}$ & $\begin{array}{c}\mathbf{d}=\mathbf{x}-\mathbf{A} \\
\mathbf{x}-\mathbf{1 2 2 . 0 9}\end{array}$ & $\mathbf{d}^{\mathbf{2}}$ & $\mathbf{f d}^{\mathbf{2}}$ \\
\hline $110-115$ & 112.5 & 16 & 1800 & 16 & -9.59 & 91.96 & 1471.48 \\
\hline $115-120$ & 117.5 & 11 & 1292.5 & 27 & -4.59 & 21.06 & 231.75 \\
\hline $120-125$ & 122.5 & 6 & 735 & 33 & 0.41 & .168 & 1.00 \\
\hline $125-130$ & 127.5 & 4 & 510 & 37 & 5.41 & 29.27 & 117.07 \\
\hline $130-135$ & 132.5 & 5 & 662.5 & 42 & 10.41 & 108.37 & 541.84 \\
\hline $135-140$ & 137.5 & 4 & 550 & 46 & 15.41 & 237.47 & 949.87 \\
\hline $140-145$ & 142.5 & 2 & 285 & 48 & 20.41 & 416.56 & 833.14 \\
\hline $145-150$ & 147.5 & 1 & 147.5 & 49 & 25.41 & 645.67 & 645.67 \\
\hline & & $\sum \mathrm{f}=49$ & $\sum \mathrm{fx}=5982.5$ & & & & $\sum \mathrm{fd}^{2}=4791.82$ \\
\hline
\end{tabular}

Mean $(A)=\sum f x / \sum f=5982.5 / 49=122.09, \operatorname{Median}(M)=$ 118.86, Mode $(\mathrm{Mo})=113.07$
Standard deviation $(\sigma)=\sqrt{ }\left(\sum \mathrm{fd}^{2} / \sum \mathrm{f}\right)=9.88$, Variance $\left(\sigma^{2}\right)$ $=97.79$ and

Co-efficient of variation $=(\sigma / \mathrm{A}) \times 100=8.09 \%$

Table 17: Frequency distribution of loading cycle time (second) at lead 25-30metres

\begin{tabular}{|c|c|c|c|c|c|c|c|}
\hline $\begin{array}{c}\text { Class } \\
\text { interval }\end{array}$ & $\begin{array}{c}\text { Class } \\
\text { mark } \\
(\mathbf{x})\end{array}$ & $\begin{array}{c}\text { Frequency } \\
(\mathbf{f})\end{array}$ & $\mathbf{f x}$ & $\mathbf{c . f}$ & $\begin{array}{c}\mathbf{d}=\mathbf{x}-\mathbf{A} \\
\mathbf{x}-\mathbf{1 5 3 . 0 9}\end{array}$ & $\mathbf{d}^{2}$ & $\mathbf{f d}^{2}$ \\
\hline $120-130$ & 125 & 7 & 875 & 7 & -28.09 & 789.05 & 5523.33 \\
\hline $130-140$ & 135 & 9 & 1215 & 16 & -18.09 & 327.25 & 2945.23 \\
\hline $140-150$ & 145 & 6 & 870 & 22 & -8.09 & 65.45 & 392.68 \\
\hline $150-160$ & 155 & 7 & 1085 & 29 & 1.91 & 3.65 & 25.54 \\
\hline $160-170$ & 165 & 2 & 330 & 31 & 11.91 & 141.85 & 283.69 \\
\hline $170-180$ & 175 & 3 & 525 & 34 & 21.91 & 480.05 & 1440.14 \\
\hline $180-190$ & 185 & 5 & 925 & 39 & 31.91 & 1018.25 & 5091.24 \\
\hline $190-200$ & 195 & 1 & 195 & 40 & 41.91 & 1756.45 & 1756.45 \\
\hline $200-210$ & 205 & 2 & 410 & 42 & 51.91 & 2694.65 & 5389.29 \\
\hline & & $\sum \mathrm{f}=42$ & $\sum \mathrm{fx}=6430$ & & & & $\sum \mathrm{fd}^{2}=22842.49$ \\
\hline
\end{tabular}

Mean $(A)=\sum f x / \sum f=6430 / 42=153.09, \operatorname{Median}(M)=$ 148.33, Mode $(\mathrm{Mo})=134$,

Standard deviation $(\sigma)=\sqrt{ }\left(\sum \mathrm{fd}^{2} / \sum \mathrm{f}\right)=23.32$, Variance $\left(\sigma^{2}\right)$ $=543.86$ and

Co-efficient of variation $=(\sigma / \mathrm{A}) \times 100=15.23 \%$
Table 18: Average loading cycle time w.r.t. different lead

\begin{tabular}{|c|c|}
\hline Lead ( metre) & Average cycle time (second) \\
\hline Upto 5 & 51.12 \\
\hline $5-10$ & 75.58 \\
\hline $10-15$ & 83.29 \\
\hline $15-20$ & 99.74 \\
\hline $20-25$ & 122.09 \\
\hline $25-30$ & 153.09 \\
\hline
\end{tabular}


Table 19: Monthly average stowing rate (te/hr) during the study period

\begin{tabular}{|c|c|c|c|c|}
\hline Date & $\begin{array}{l}\text { Daily Stowing } \\
\text { rate (te/hr.) }\end{array}$ & Date & $\begin{array}{c}\text { Daily Stowing } \\
\text { rate (te/hr.) }\end{array}$ & $\begin{array}{c}\text { Monthly average } \\
\text { stowing rate (te/hr) }\end{array}$ \\
\hline $2 / 4 / 2006$ & 105 & $2 / 5 / 2006$ & 87.5 & \multirow{12}{*}{$\begin{array}{c}(90.95+96.05) / 2 \\
=93.5\end{array}$} \\
\hline $3 / 4 / 2006$ & 92.85 & $3 / 5 / 2006$ & 100 & \\
\hline $5 / 4 / 2006$ & 89.65 & $4 / 5 / 2006$ & 100 & \\
\hline $10 / 4 / 2006$ & 94.16 & $8 / 5 / 2006$ & 100 & \\
\hline $11 / 4 / 2006$ & 90 & $9 / 5 / 2006$ & 90.9 & \\
\hline $14 / 4 / 2006$ & 57.14 & $10 / 5 / 2006$ & 95 & \\
\hline $17 / 4 / 2006$ & 90.47 & $11 / 5 / 2006$ & 85.71 & \\
\hline $19 / 4 / 2006$ & 100 & $18 / 5 / 2006$ & 100 & \\
\hline $24 / 4 / 2006$ & 88.88 & $19 / 5 / 2006$ & 97.43 & \\
\hline $25 / 4 / 2006$ & 92.31 & $20 / 5 / 2006$ & 100 & \\
\hline $26 / 4 / 2006$ & 100 & $28 / 5 / 2006$ & 100 & \\
\hline $\begin{array}{l}\text { Monthly daily } \\
\text { average stowing rate } \\
\text { (te/hr.) }\end{array}$ & 90.95 & & 96.05 & \\
\hline
\end{tabular}

Table 20: Average intake quantity, Quantity at LVC and VEQ (\%)

\begin{tabular}{|c|c|c|c|}
\hline $\begin{array}{c}\text { Measurement } \\
\text { Date }\end{array}$ & $\begin{array}{c}\text { Intake quantity } \\
\text { (cu.m /min.) }\end{array}$ & $\begin{array}{c}\text { Quantity at LVC } \\
\text { (cu.m /min.) }\end{array}$ & VEQ (\%) \\
\hline $4 / 10 / 2005$ & 1017 & 763 & 75 \\
\hline $31 / 10 / 2005$ & 1067 & 610 & 57.16 \\
\hline $5 / 11 / 2005$ & 1050 & 720 & 68.57 \\
\hline $24 / 11 / 2005$ & 1024 & 694 & 67.77 \\
\hline $2 / 12 / 2005$ & 1008 & 696 & 70.48 \\
\hline $16 / 12 / 2005$ & 1064 & 750 & 71.15 \\
\hline $4 / 01 / 2006$ & 1085 & 772 & 72.06 \\
\hline $18 / 01 / 2006$ & 1117 & 805 & 72.11 \\
\hline $4 / 02 / 2006$ & 1101 & 794 & 82.26 \\
\hline $18 / 02 / 2006$ & 902 & 742 & 75 \\
\hline $7 / 3 / 2006$ & 1113 & 834.75 & 64.26 \\
\hline $16 / 3 / 2006$ & 746 & 554 & 90.9 \\
\hline $4 / 4 / 2006$ & 763 & 534 & 94.75 \\
\hline $2 / 4 / 2006$ & 931 & 847 & 74.03 \\
\hline $15 / 5 / 2006$ & 706 & 669 & \\
\hline Average & 979.6 & 718.98 & \\
\hline
\end{tabular}

\section{RESULTS \& DISCUSSION}

\subsection{Results of Various Operations}

Results of various operations of face preparation sub-system \& loading sub-system are plotted as frequency distribution curves, and, shown in fig. 3to 17. Frequency distribution curves have been drawn from table 3 to 17 . From these frequency distribution curves various time elements have been estimated and used for the computation of system capacities.

Further, in order to estimate the average loading cycle time (by SDLs at various lead distances) the SDL loading time was studied in the field \& the results are graphically plotted as shown in fig. 18. Graph has been plotted from table 18.

Again, in order to estimate the average stowing rate the stowing operation was studied in the field \& the results are graphically plotted in fig. 19. Graph has been plotted from table 19.

Again, in order to estimate the intake quantity, quantity at LVC \& VEQ (\%) ventilation operation was studied in the field $\&$ the results are graphically plotted in fig. 20. Graph has been plotted from table 20 . 


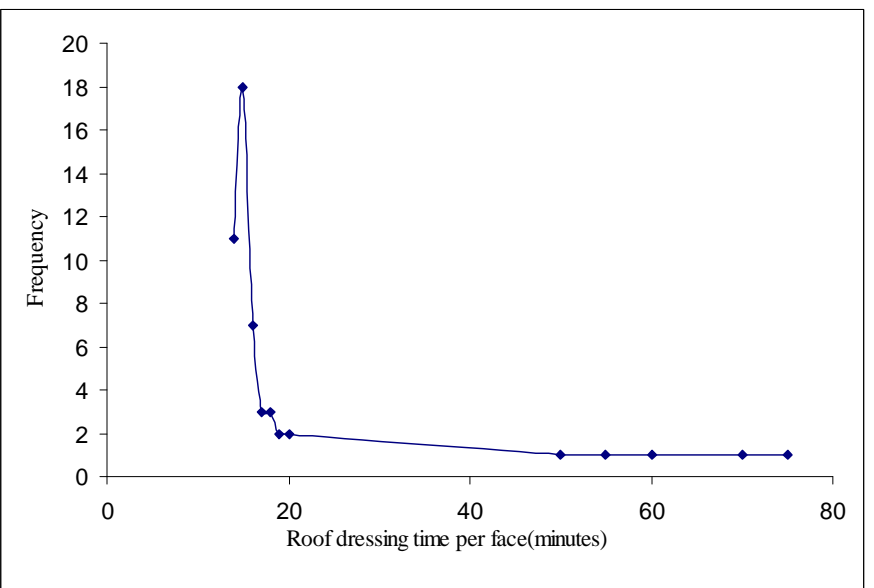

Fig. 3: Roof dressing time per face vs. Frequency

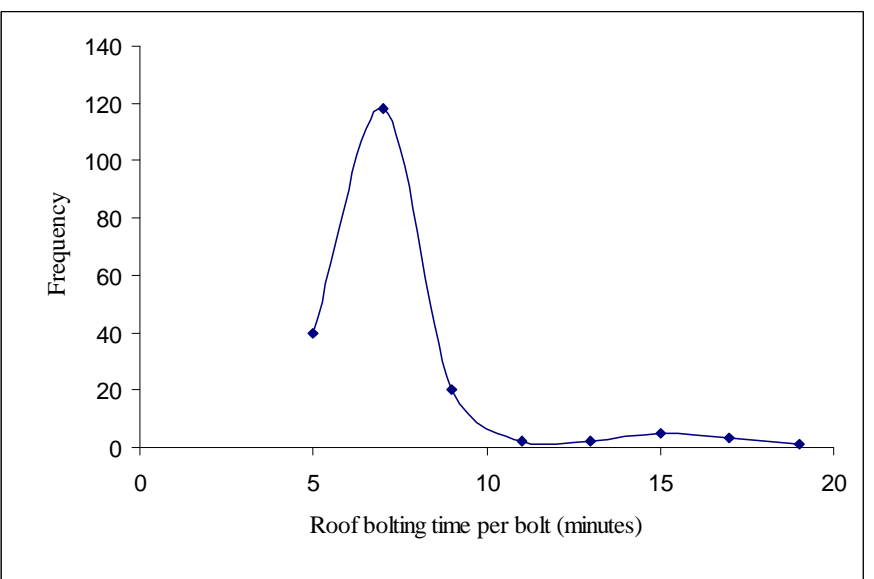

Fig. 4: Roof bolting time per bolt vs. Frequency

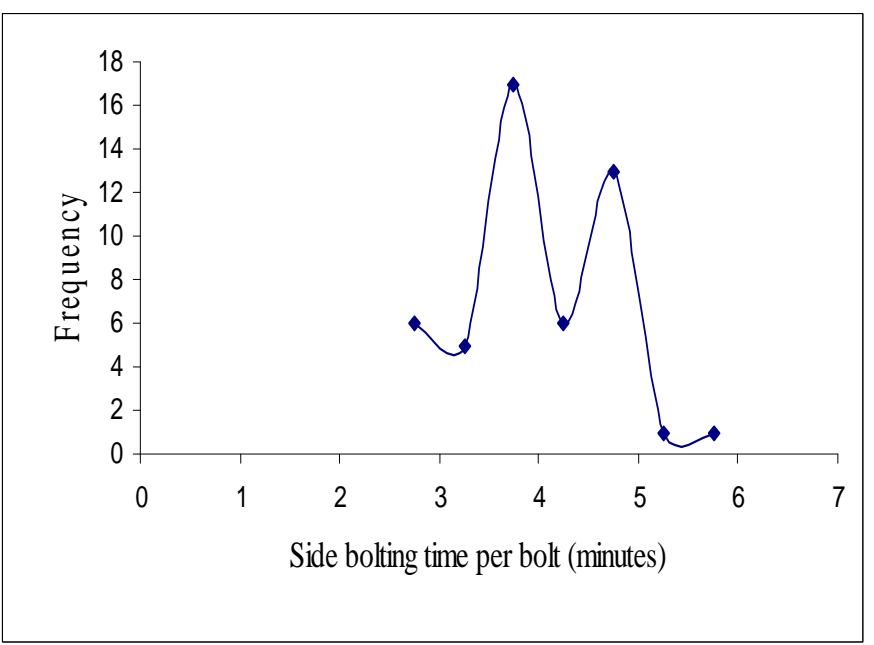

Fig. 5: Side bolting time per bolt vs. Frequency

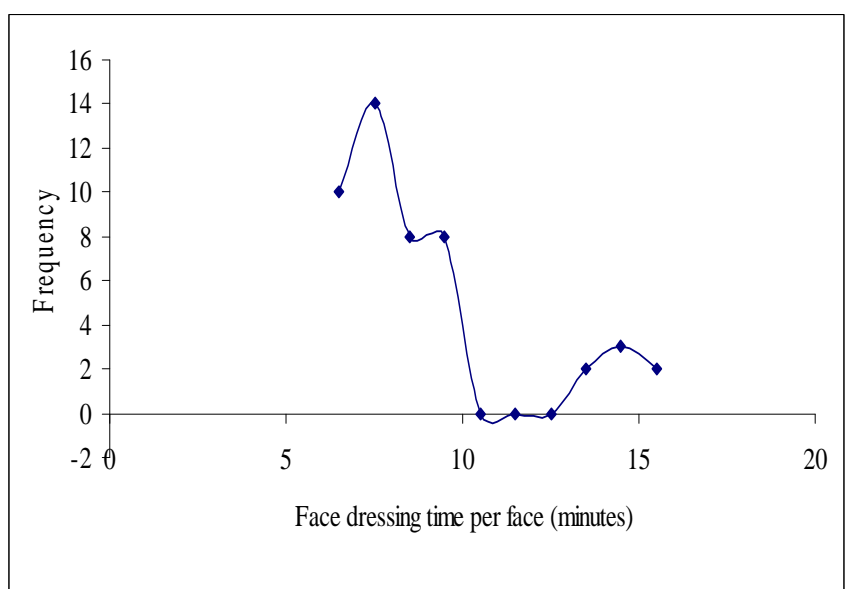

Fig. 6: Face dressing time per face vs. Frequency

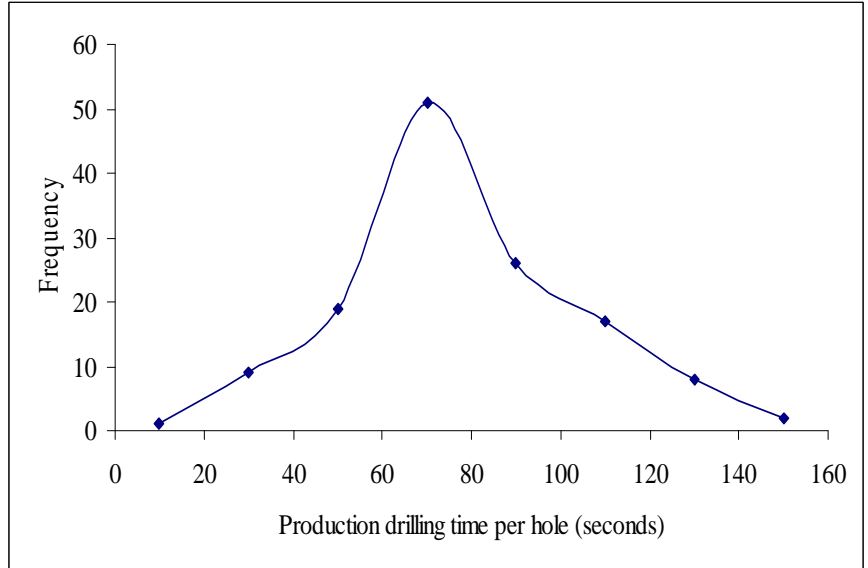

Fig. 7: Production drilling time per hole vs. Frequency

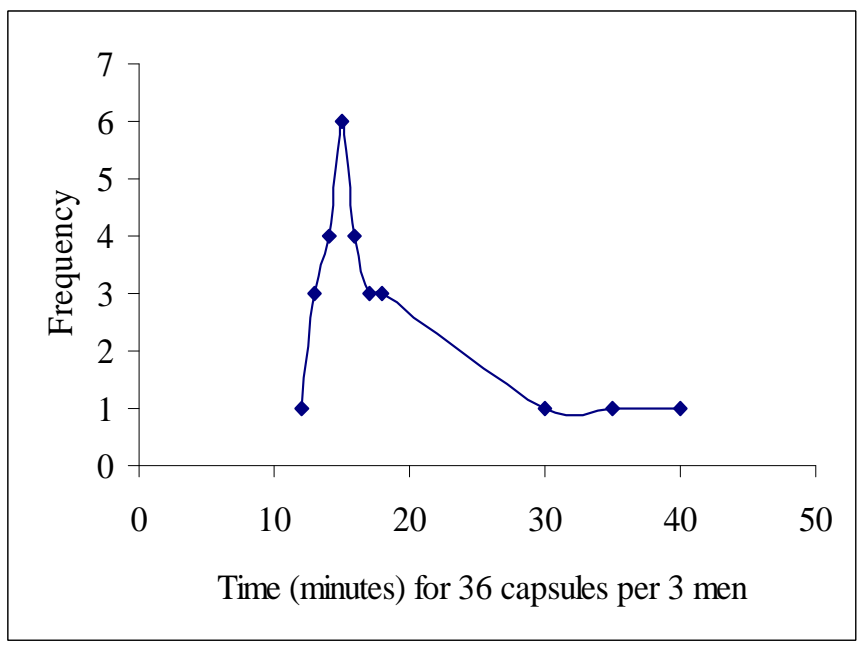

Fig. 8: Time for 36 sand capsules per 3 men vs. Frequency 


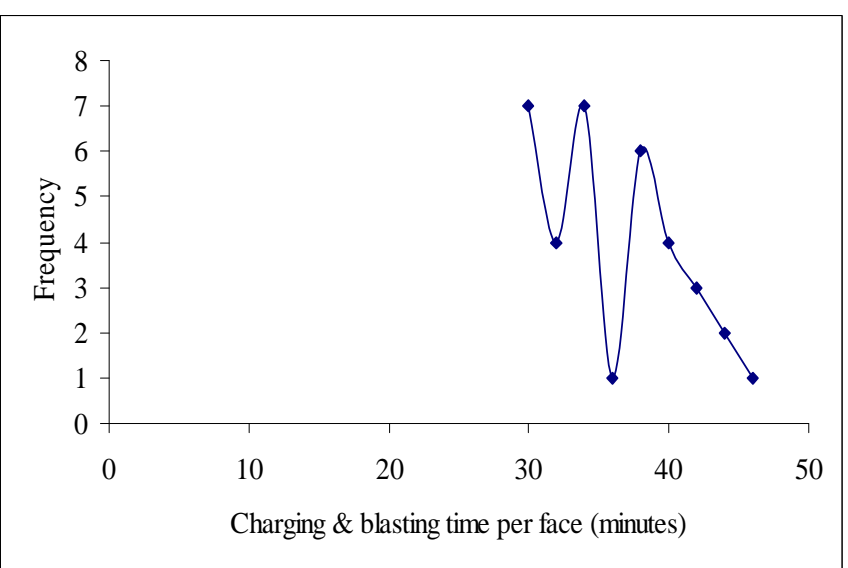

Fig. 9: Charging and blasting time per face vs. Frequency

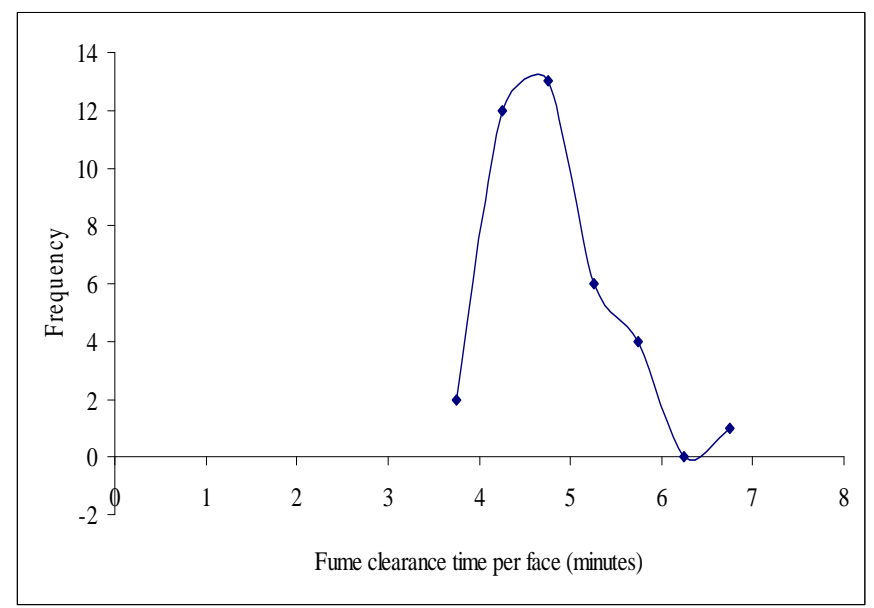

Fig. 10: Fume clearance time per face vs. Frequency

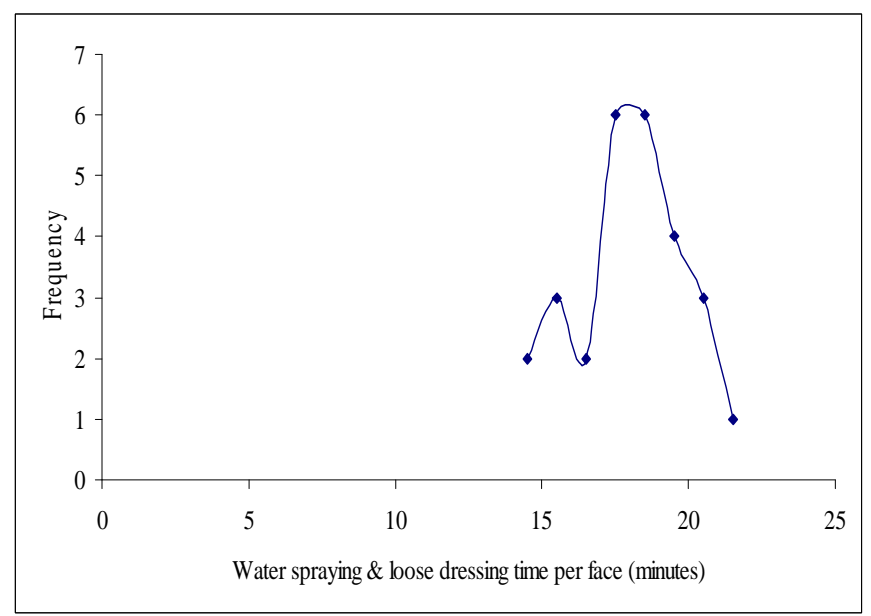

Fig. 11: Water spraying \& loose dressing time per face vs. Frequency

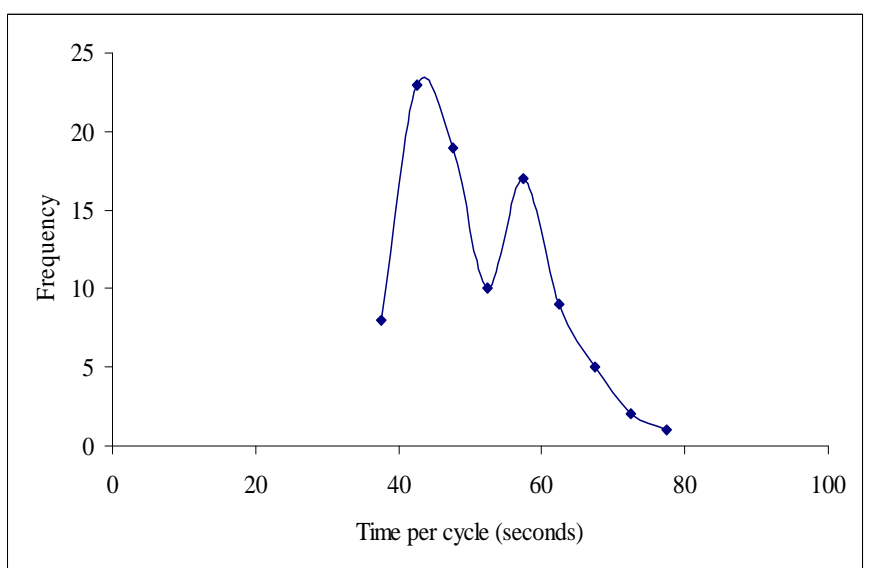

Fig. 12: Loading cycle time (lead up to $5 \mathrm{~m}$ ) vs. Frequency

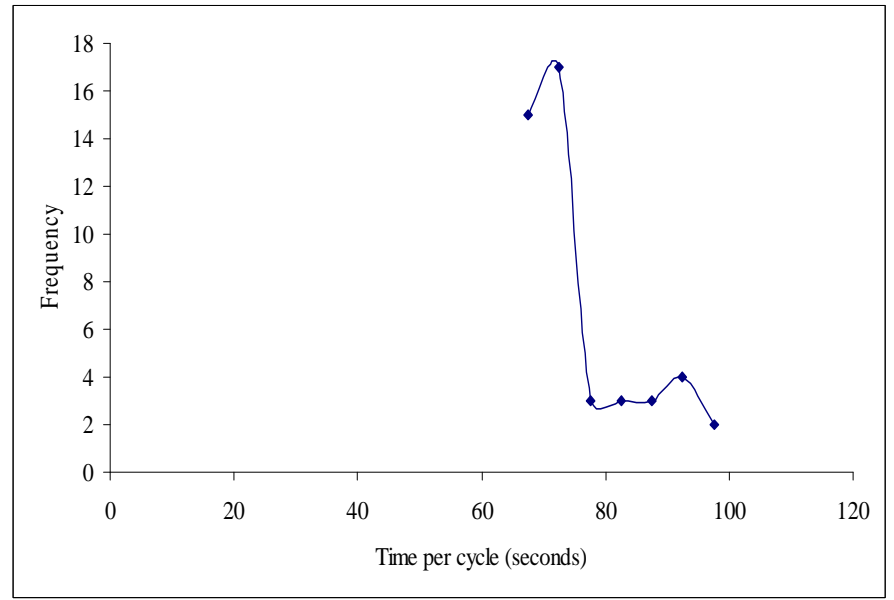

Fig. 13: Loading cycle time (lead 5-10m) vs. Frequency

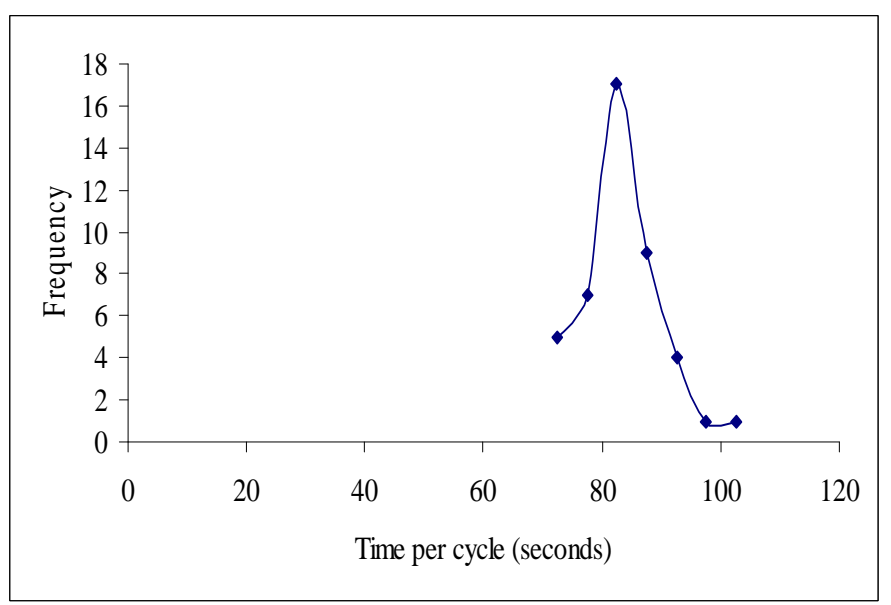

Fig. 14: Loading cycle time (lead 10-15m) vs. Frequency 


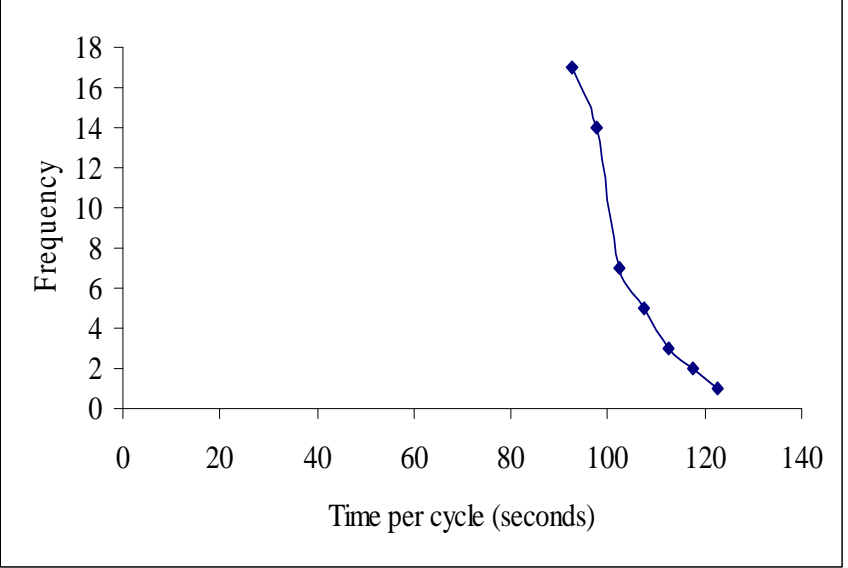

Fig.15: Loading cycle time (lead 15-20m) vs. Frequency

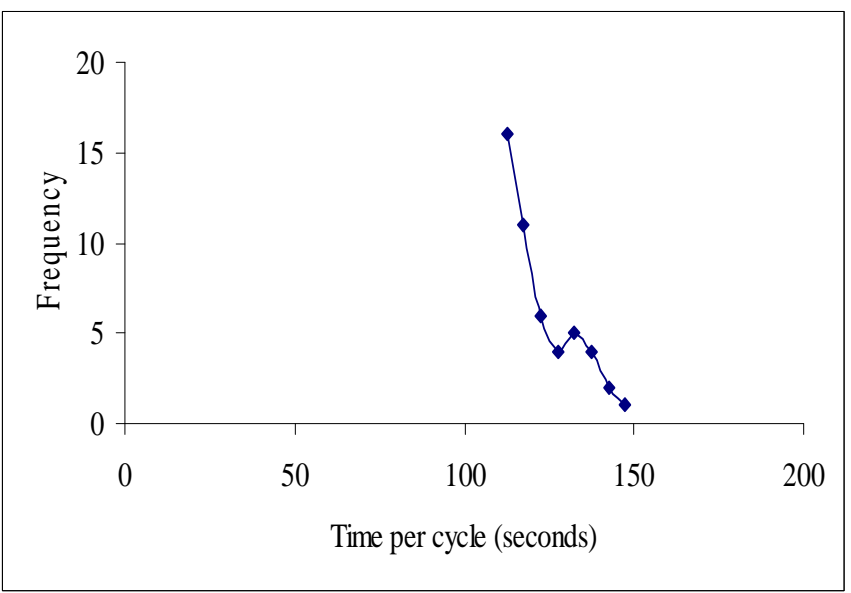

Fig. 16: Loading cycle time (lead 20-25m) vs. Frequency

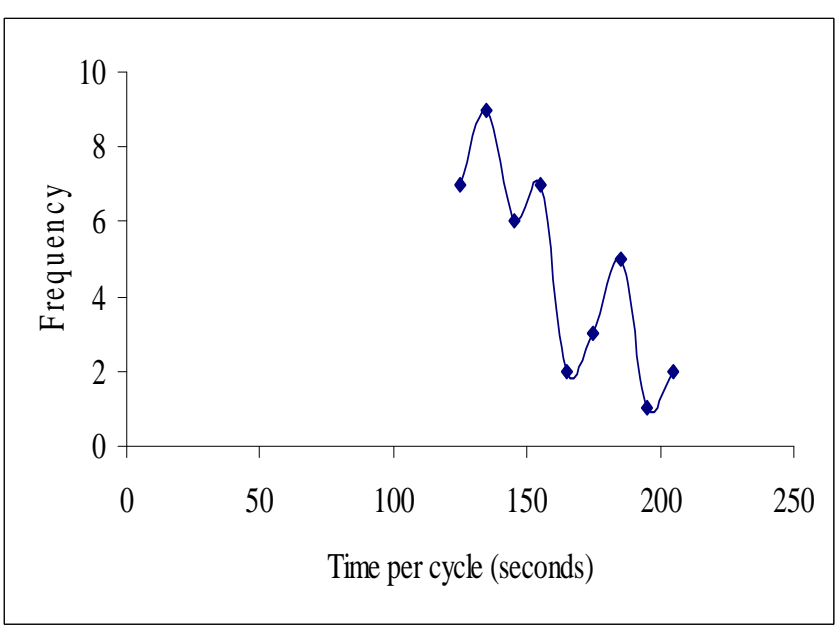

Fig. 17: Loading cycle time (lead 25-30m) vs. Frequency

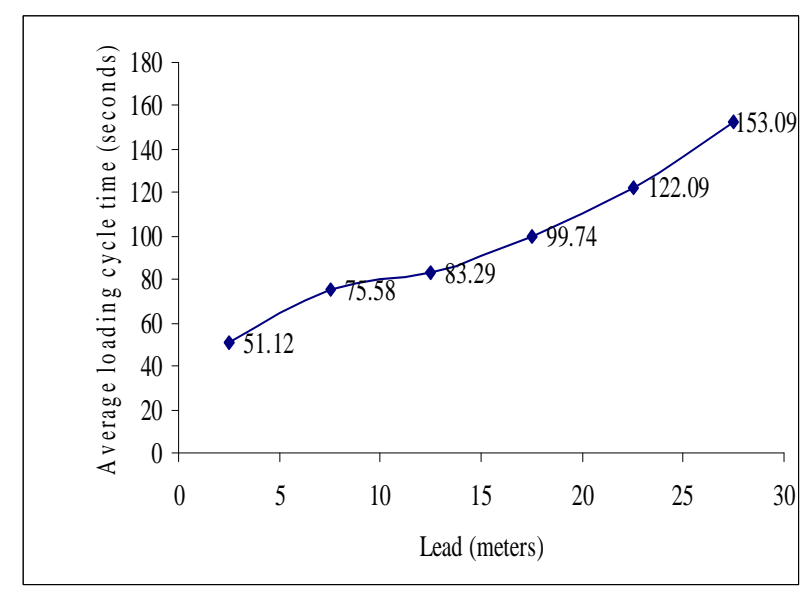

Fig. 18: Lead vs. Average loading cycle time

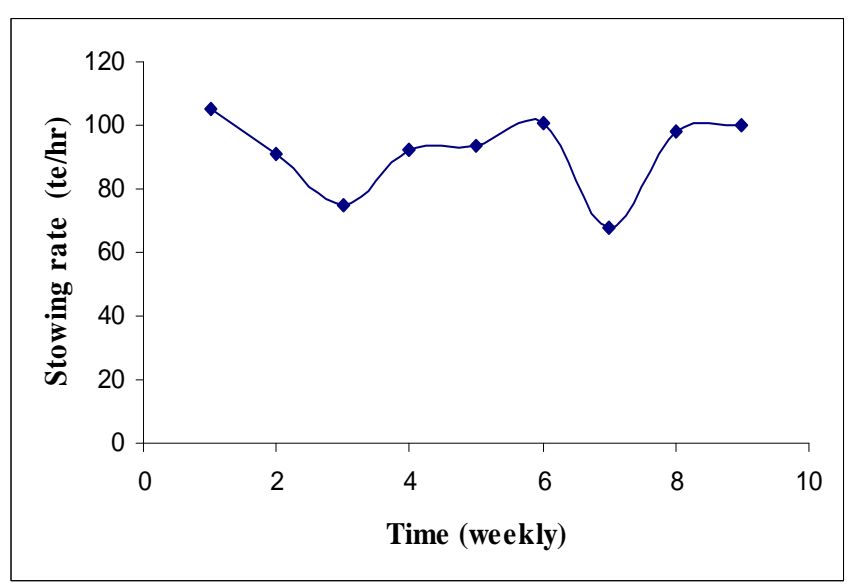

Fig. 19: Time vs. stowing rate

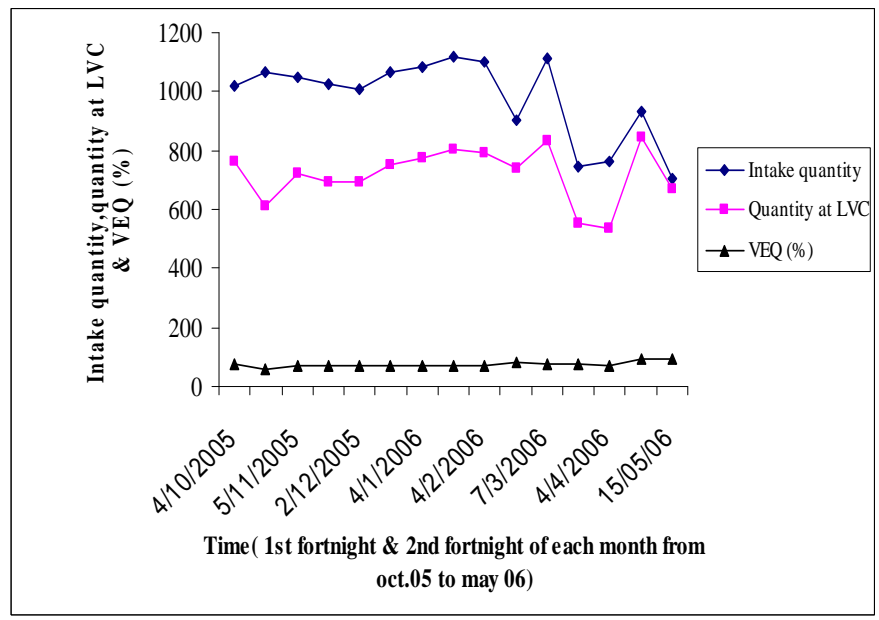

Fig. 20: Time vs. Intake quantity (cu.m/min.), Quantity at LVC and VEQ (\%) 


\subsection{Statistical Results of Face Preparation \& Loading}

\section{Sub-System:}

Statistical results of face preparation \& loading sub-system are tabulated in table 21. Results in this table have been drawn from frequency distribution table 3 to 17 (Except table 8)

Table 21: Integrated table showing the statistical results of face preparation and loading sub-systems

\begin{tabular}{|c|c|c|c|c|c|c|}
\hline Operations & Mean & Median & Mode & $\begin{array}{l}\text { Standard } \\
\text { Deviation }\end{array}$ & Variance & $\begin{array}{c}\text { Co-efficient } \\
\text { of variation } \\
(\%)\end{array}$ \\
\hline \multicolumn{7}{|l|}{ face preparation time } \\
\hline 1. Roof dressing per face(minutes) & 20.18 & 15 & 15 & 14.17 & 200.92 & 70.24 \\
\hline 2. Roof bolting per bolt (minutes) & 7.32 & 6.94 & 6.88 & 2.38 & 5.69 & 32.51 \\
\hline 3.Side bolting per bolt(minutes) & 3.97 & 3.89 & 3.76 & 0.736 & 0.542 & 18.53 \\
\hline 4.Face dressing per face(minutes) & 8.84 & 7.96 & 7.4 & 2.57 & 6.65 & 29.16 \\
\hline 5.Production drilling per hole (seconds) & 80 & 74.7 & 71.23 & 27.04 & 731.57 & 33.7 \\
\hline 6.Charging \& blasting per face (minutes) & 36 & 34.85 & 33.67 & 4.68 & 21.94 & 13 \\
\hline 7.Fume clearance per face(minutes) & 4.78 & 4.69 & 4.56 & .616 & .381 & 12.88 \\
\hline 8.WS+LD per face(minutes) & 17.98 & 18.08 & 18 & 3.31 & 10.93 & 18.41 \\
\hline \multicolumn{7}{|l|}{ Loading cycle time (seconds) } \\
\hline 1 Lead $(0-5 m)$ & 51.12 & 49.21 & 43.95 & 9.38 & 87.98 & 18.34 \\
\hline 2. Lead $(5-10 \mathrm{~m})$ & 75.58 & 72.5 & 70.63 & 9.02 & 81.44 & 11.94 \\
\hline 3.Lead (10-155m) & 83.29 & 82.94 & 82.77 & 6.57 & 43.11 & 7.88 \\
\hline 4.Lead (15-20m) & 99.74 & 97.67 & 94.25 & 7.07 & 50.06 & 7.09 \\
\hline 5.Lead $(20-25 \mathrm{~m})$ & 122.1 & 118.86 & 113.07 & 9.88 & 7.79 & 8.09 \\
\hline 6.Lead (25-30m) & 153.1 & 148.33 & 134 & 23.321 & 543.86 & 15.23 \\
\hline
\end{tabular}

From the analysis of the results as given in table 21 the following important discussion may be drawn:

Statistical analysis of roof dressing operation shows significantly high value of co-efficient of variation $(70.24 \%)$ in comparison to other operations which shows that data is not consistent. This, in turn, implies that the roof dressing operation is inconsistent in nature, being dependent and the prevailing geo-mining conditions. In this panel, particularly, the roof conditions were bad \& variable due to presence of carbonaceous shale.

\subsection{Results for Capacity Estimation:}

From the fore going table $21 \&$ related statistical computation (mean, median, mode, standard deviation \& co-efficient of variation), it is quite justified to use the mean time element for the purpose of capacity estimation. Median \& Mode appears to provide very optimistic data in uncertain geo-mining conditions. That is why the mean appears justifiable.

Table $22 \& 23$ provides the mean time element for various sub-systems under study.
Table 22: Mean time elements for various operations used for capacity estimation of sub-systems (Face Preparation \& Loading)

\begin{tabular}{|l|c|}
\hline \multicolumn{1}{|c|}{ Operations } & $\begin{array}{c}\text { Statistical } \\
\text { Time } \\
\text { (mean) }\end{array}$ \\
\hline Face preparation time: & \\
\hline 1. Roof dressing per face(minutes) & 20.18 \\
\hline 2. Roof bolting per bolt(minutes) & 7.32 \\
\hline 3.Side bolting per bolt(minutes) & 3.97 \\
\hline 4.Face dressing per face(minutes) & 8.84 \\
\hline 5.Production drilling per hole (seconds) & 80 \\
\hline $\begin{array}{l}\text { 6. Charging \& blasting per face } \\
\text { (minutes) }\end{array}$ & 36 \\
\hline 7.Fume clearance per face(minutes) & 4.78 \\
\hline $\begin{array}{l}\text { 8. Water spraying \& Loose dressing per } \\
\text { face (minutes) }\end{array}$ & 17.98 \\
\hline Loading cycle time (seconds): & \\
\hline 1. Lead (15-20m) & 99.74 \\
\hline
\end{tabular}


Table 23: Mean time elements for various operations used for capacity estimation of sub-systems (ventilation, transportation \& stowing)

\begin{tabular}{|c|c|c|}
\hline Ventilation sub-system & Transportation sub-system & Stowing sub-system \\
\hline $\begin{array}{c}\text { Average intake quantity } \\
=979.6 \mathrm{cu} . \mathrm{m} / \mathrm{min} .\end{array}$ & Tipper belt= $20 \mathrm{hrs} . /$ day & $\begin{array}{c}\text { Average stowing rate } \\
93.5 \mathrm{te} / \mathrm{hr}(\text { on monthly basis) }\end{array}$ \\
\hline $\begin{array}{c}\text { Average quantity at LVC } \\
=718.98 \mathrm{cu} . \mathrm{m} / \mathrm{min} .\end{array}$ & Trunk belt= $20 \mathrm{hrs} . / \mathrm{day}$ & \\
\hline $\begin{array}{c}\text { Average leakages quantity } \\
=260.62 \text { cu.m/min. }\end{array}$ & Sectional belt $=18 \mathrm{hrs} . /$ day & \\
\hline & Face chain conveyor $=18 \mathrm{hrs} . /$ day & \\
\hline
\end{tabular}

\section{CONCLUSIONS}

1. Statistical analysis of roof dressing operation shows significantly high value of co- efficient of variation which means roof condition is not good.

2. Mean time elements will be considered as standard time for Capacity estimation purpose of various SubSystems

\section{REFERENCES}

[1] Bhattcherjee, A., Maiti, J. and Mukherjee, N. "An investigation of system performance of mechanized bord-and-pillar mine working through discrete event simulation modeling", Minetech Journal., Vol. 17, No.6, Nov.-Dec.,1996, pp.33-37.

[2] Chaoji, S.V. "Environmental challenges and future of Indian coal", Journal of Mines, Metals and Fuels, Vol. 50, No.7, July, 2002, pp.257-261

[3] Karmakar, H.N. "New generation mine modeling for higher production and productivity of underground coal mines", Minetech Journal, Vol. 17, No.6, Nov.Dec.,1996, pp. 39-42.

[4] Mathur, S.P. "Labour productivity and cost reduction in Indian coal mines". Minetech Journal, Vol.11, No. 3, May-June, 1999, pp. 5-9

[5] Mukhopadhyay, S.B. and Babu, A.V. "System analysis approach in mining operations-an overview", Minetech Journal,Vol.14, No.5-6, Sep-Dec.,1993. pp.30-36.

[6] Rai,P. \& Singh, Arvind Kumar. "System analysis approach for production planning in underground coal mine", Minetech, Vol.26, No.2, Mar- Apr.,2005, pp.2328

[7] Rai, P. "Some aspects of material transportation by rear dump trucks in opencast mines", Minetech Journal, Vol.22, No.3, May-Jun., 2001, pp.3-10.

[8] Ray, S.C. And Sinha, D.K. "Capacity analysis of mine system-a pre-requisite for production planning in underground mines", Indian Mining \& Engineering Journal, Vol.17., No.5, May,1978, pp.7-12.
[9] Sachdev, B.K. "Coal industry in India development issues" Procs. 4th Asian mining international conference on geology, mining and mineral processing. Nov. 24-28, Calcutta, 1993. 